\title{
Incremental Dynamic Semantics for Language-based Programming Environments
}

\author{
Gail E. Kaiser \\ Carnegie Mellon University
}

\begin{abstract}
Attribute grammars are a formal notation for expressing the static semantics of programming languages - those properties that can be derived from inspection of the program text. Attribute grammars have become popular as a mechanism for generating language-based programming environments that incrementally perform symbol resolution, type checking, code generation and derivation of other static semantic properties as the program is modified. However, attribute grammars are not suitable for expressing dynamic semantics - those properties that reflect the history of program execution and/or user interactions with the programming environment. This article presents action equations, an extension of attribute grammars suitable for specifying the static and the dynamic semantics of programming languages. It describes how action equations can be used to generate language-based programming environments that incrementally derive static and dynamic properties as the user modifies and debugs the program.
\end{abstract}

Categories and Subject Descriptors: D.2.3 [Software Engineering]: Coding - program editors; D.2.5 [Software Engineering]: Testing and Debugging - debugging aids; D.2.6 [Software Engineering]: Programming Environments; D.3.1 [Programming Languages]: Formal Definitions and Theory - semantics; D.3.4 [Programming Languages]: Processors interpreters, translator writing systems and compiler generators; F.3.1 [Logics and Meanings of Programs]: Specifying and Verifying and Reasoning about Programs - specification techniques; F.3.2 [Logics and Meanings of Programs]: Semantics of Programming Languages - operational semantics

General Terms: Algorithms, Design, Experimentation, Languages

Additional Key Words and Phrases: Attribute grammars, dynamic semantics, generation of language-based environments, interpreters

This research was supported by a Fannie and John Hertz Foundation Fellowship, by the United States Army, Software Technology Development Division of CECOM COMM/ADP, Fort Monmouth, NJ and by ZTI-SOF of Siemens Corporation, Munich, Germany.

Author's current address: Columbia University, Department of Computer Science, New York, NY 10027. 


\section{Introduction}

This article addresses the processing performed by language-based environments (LBEs). This processing is performed automatically and incrementally (in the background) as the user writes and tests his programs. It requires an internal representation that consists of the program itself plus additional information maintained by the environment during program construction and execution. This information represents two kinds of semantic properties, static and dynamic. Static properties are those that can be determined by inspection of the program, while dynamic properties reflect the interaction between the user and the environment. The implementor of an LBE describes its processing as derivation and manipulation of these properties. For example, symbol resolution, type checking and code generation involve static properties, while interpretation, run-time support and symbolic debugging involve dynamic properties.

Recent research has focused on the generation of LBEs from descriptions. Several mechanisms have been proposed for specifying the processing performed by the environments, and the most successful of these have been action routines, attribute grammars and denotational semantics. Action routines are written as a collection of imperative subroutines. Consequently, it has proved difficult for an implementor of an environment to anticipate all possible interactions among these subroutines that may result in adverse behavior. Attribute grammars are written in a declarative style and the implementor need not be concerned with subtle interactions because all interactions among semantic equations can be determined automatically. Attribute grammars have been successfully applied only to the description of static semantics, and have hitherto seemed unsuited to the description of dynamic semantics. Denotational semantics is a formal mechanism that provides direct means for defining certain dynamic properties, notably interpretation. Denotational semantic specifications have not been extended to other dynamic processing such as interactive debugging nor to incremental detection and reporting of static semantic errors.

This article proposes an extension to attribute grammars that supports incremental processing of both static and dynamic semantics. The extended paradigm is called action equations. Action equations are written in a notation that retains the flavor of attribute grammars but adds an easy means to express dynamic properties as well as static properties. The extensions to attribute grammars include attaching particular semantic equations to events that represent user commands and supporting dependencies among events as well as among attribute values. The 
applicative nature of attribute grammars is relaxed, allowing attributes to be treated as variables and permitting modification in addition to replacement for changing the values of attributes. Together, these extensions are sufficient to support incremental processing of dynamic semantics.

\section{Generation of Language-Based Environments}

LBEs are an alternative to the traditional tools used by programmers to edit, compile and debug their programs. The key components of an LBE are a standard user interface and a common program representation. Many programming environments have been built using structure editing technology, which supports both of these features. The user interface consists of some mixture of template editing and text editing (supported by incremental parsing $[23,35,63])$; the program is represented as a parse tree or abstract syntax tree, where each node may be decorated with attributes. Some of the best known LBEs are Mentor [11], Interlisp [60], the Program Synthesizer [59], Gandalf [24], Pecan [50], and Rational [2]. Each of these environments consists of an integrated collection of tools that (1) can be viewed as a single tool [7] and (2) may be applied incrementally as the programmer writes and tests his programs. In some cases, the tools are automatically applied without explicit intervention by the programmer. For example, type checking and symbol resolution may be performed automatically as the program is created and modified; code generation and some code optimization may also be done incrementally.

The early LBEs were hand-coded. Then several environment generators were developed, including ALOE [45], Metal [12] and the Synthesizer Generator [53]. An environment generator is a program that combines an environment description with the editor kernel to produce the desired LBE. The editor kernel provides the facilities common to all environments, such as window management and language-independent tree manipulation commands, while the environment description includes all the information specific to the desired programming environment. The person who writes the environment description is called the implementor of the environment while a person who uses the environment to write his programs is called a user.

An environment description has two components, the syntax description and the semantics description. The syntax description includes the abstract syntax of the programming language and the user interface (or concrete syntax) for the language. This information is normally 
provided as some form of context-free grammar. A syntax description alone is sufficient as an environment description if no semantics processing is required. An environment generator can combine the syntax description with the editor kernel to produce a pure syntax-directed editor that supports program editing and enforces correct syntax.

\subsection{Semantics Description}

The semantics description specifies all the processing performed by the environment, i.e., everything the environment does that is not among the standard facilities provided by the editor kernel. Although an LBE is a single tool, the semantics processing of an LBE is performed by what is conceptually a collection of tools and tool fragments that are knowledgeable about the particular programming language. The collection can be subdivided into tools that handle static semantics and tools that handle dynamic semantics.

The static semantics of a program involve those properties that, by definition, cannot change during its execution. The static properties of a conventional, lexically scoped programming language include symbol resolution, type identification and the object code generated for the program. For example, the set of identifiers defined in a particular program, the mapping between identifier uses and identifier definitions, and the types assigned to particular identifiers and expressions are all in the realm of static semantics.

Consider the program fragment in figure 2-1. The program states that the variable $\mathbf{a}$ is declared to be of type integer, but the program also states that the variable a constitutes the conditional expression of the if statement. The static semantics of this programming language require that a variable has the same type over its lifetime and that the condition expression of every if statement is of type boolean. Thus there is a static semantic error in the program. A programming environment that included a type checking tool would warn the user of this error.

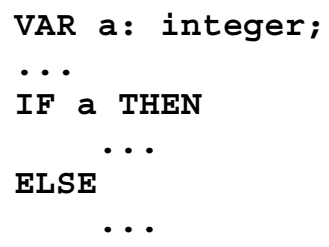

Figure 2-1: Type Checking Example

If the tool were incremental, it would warn the user as soon as the error could be detected. If 
the user had first entered that the type of $\mathbf{a}$ is integer, and later used $\mathbf{a}$ as the conditional expression for the if statement, then the error would be detected and reported immediately after the user entered the conditional expression. If the user had instead added a to the list of variables, without indicating its type, then used a as the conditional expression, and finally returned to the variables list to state that $\mathbf{a}$ is an integer, then the error would be caught immediately after the user entered this type information.

The dynamic semantics of a program involves the derivation and manipulation of those properties that may change during the execution of the program. The dynamic properties of a conventional language include the assignment of values to particular storage locations and the maintenance of the current focus of execution behavior (i.e., the program counter). The area of dynamic semantics includes run-time support and symbolic debuggers as well as interpreters.

The same programming environment sketched above might include an interpreter as well as the type checking tool. The job of the interpreter is to directly execute programming language statements. The interpreter does not need to perform type checking or other static semantics processing, since these functions are handled by other tools. The interpreter performs the activities that the program fragment is defined to do according to the dynamic semantics of the programming language.

Consider the corrected program fragment in figure 2-2. The interpreter would begin execution of the if statement by getting the current value of the a variable from the store (which binds variable locations to values). If a does not have a value, this would be reported to the user as an error (or the environment could ask the user to enter a value). If a does have a value, the interpreter would then check whether it is 'true' or 'false'. If true, the interpreter would execute the then statement; if false, it would execute the else statement.

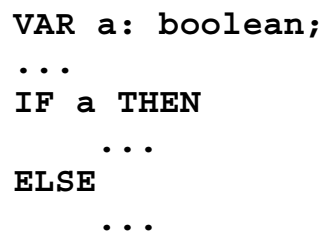

Figure 2-2: Interpretation Example

This behavior does not depend on whether the interpreter tool is incremental or non- 
incremental. By analogy to the type checking tool, an 'incremental' interpreter might follow along behind the user, executing the program as it is typed, as in VisiProg [25]. Instead, we think of an 'incremental' interpreter as one that permits the user to select, for example, the then part of the if, the entire if statement, or an arbitrary program unit, and give a command to interpret that unit. In a non-incremental environment, the user would have no choice but to commence execution of the program at the beginning.

Specifying static and dynamic semantics is very complex. In contrast to the syntax description, there is no commonly accepted paradigm for the semantics description of a programming environment. There are two major schools that support different methods of specifying the semantics processing of an LBE: action routines and attribute grammars. Both methods support interactive semantics processing, i.e., the integrated, incremental, nonsequential, structure-oriented computing style described by Notkin in his thesis [46]. Such interaction with the user is an essential requirement for modern programming environments. A third major school - denotational semantics - disagrees with this claim, and supports another method of specifying semantics processing for non-incremental, sequential programming environments. These three methods are briefly described here and are explained in detail in the references.

The first school uses action routines, which were proposed by Medina-Mora in his thesis [45] for use in LBEs $[18,24]$. Action routines are based on the semantic routines used in compiler generation systems such as Yacc [31]. The semantics processing is written as a set of routines in either a conventional programming language or in a special purpose programming language designed for writing action routines [1]. A set of routines is associated with each production in the abstract syntax, one for each user command (such as Create, Delete, Enter, Exit, Execute, etc.) that can be applied to an instance of that production. The corresponding routine is automatically invoked by the editor kernel when an editing command is applied to a node in the syntax tree representing the program.

The second major group uses attribute grammars, which were introduced by Knuth [43] for specifying the context-sensitive properties of programming languages. Attribute grammars are an alternative to semantic routines in compiler-compilers $[14,20]$. The generation of LBEs from attribute grammars [32, 53] was proposed by Demers, Reps and Teitelbaum [8]. The semantics of the programming language are written as (1) a set of attribute declarations associated with 
each symbol; and (2) a collection of semantic equations - each associated with a particular production - that define the values of the attributes of the symbols on the production's left and right hand sides. The values of the attributes are determined by evaluating all the semantic equations as a set of simultaneous equations. During program editing, an incremental algorithm $[33,52]$ automatically reevaluates those attributes whose values may have changed as the result of a subtree replacement (editing operation).

The third school uses denotational semantics, originally promoted by Scott and Strachey [55] for formal reasoning about programs. The semantics of the programming language are written as a set of formal definitions - associated with each production in the abstract syntax that specify the denotation of each language construct in terms of the environment (which binds variable identifiers to locations), the store, and the denotations of other productions. Several research groups have applied denotational specifications to generation of compilers [6, 48, 49] and interpreters [5], but none of these systems are effective in an incremental programming environment. However, Johnson has recently developed an incremental interpreter/debugger for GL [34], an expressional language based on denotational semantics.

Other methods have been proposed (e.g., $[3,10,13,51])$, but none fulfill all the requirements of an LBE. The basic problems are:

- The design, implementation and debugging of action routines, or any other procedural mechanism, is tedious and error-prone compared to the ease with which a syntax description can be developed.

- The capabilities of attribute grammars, denotational specifications and the various other declarative methods are generally limited to a relatively small subset of the processing performed by modern programming environments.

This article describes a new method, action equations, that augments attribute grammars with mechanisms taken from action routines. The 'action' of action equations comes from association of user commands (or actions) with action routines, while the 'equations' comes from the semantic equations of attribute grammars. Action equations achieve a synthesis with most of the advantages of both paradigms but few of their disadvantages. Action equations were originally presented in the author's thesis [36], and additional details can be found there. 


\subsection{Overview of Action Equations}

Attribute grammars are not suitable for the description of dynamic semantics because of the inherently static nature of their attributes. The value of each attribute is equated to a specified function of the program text and other attributes. It cannot depend in any way on the history of modifications to the program text or of the execution of the program. By definition, attribute grammars are inappropriate for expressing dynamic semantics.

The primary contribution of the action equations paradigm is that it supports the expression of history or dynamic properties in a style based on attribute grammars. This is done by embedding rules similar in form to semantic equations in an event-driven architecture. Events correspond to user commands and activate their attached equations in the same sense that, in the action routines paradigm, commands trigger the associated action routines. The editor kernel orders the evaluation of active equations according to the commands invoked by the user and the dependencies among attributes and events as defined by the equations. Equations that apply at all times are not attached to particular events and these correspond exactly to the semantic equations of attribute grammars.

Those action equations attached to events, however, should not be confused with semantic equations. Attribute grammars are applicative: an attribute is a variable in the sense of algebra's simultaneous equations but not in the sense of conventional programming languages. An attribute is reevaluated only when the program is modified, and then the semantic equation replaces the old value with an entirely new value.

These restrictions are relaxed for action equations, as follows. First, an equation may be reevaluated due to the selection of an event, so an attribute may be reassigned many times even though the program has not changed. Second, an equation is permitted to define the new value of the attribute as a modification of its previous value in the case of aggregate (or composite) values, such as the symbol table and the run-time stack. This second extension to pure attribute grammars has recently appeared in several 'attribute grammar' systems [26, 54]. Together, these side-effects and the added dimension of events make it possible for action equations to support the expression of dynamic semantics in a style similar to how attribute grammars support the expression of static semantics. 


\section{Description of Dynamic Semantics}

\subsection{Action Equations}

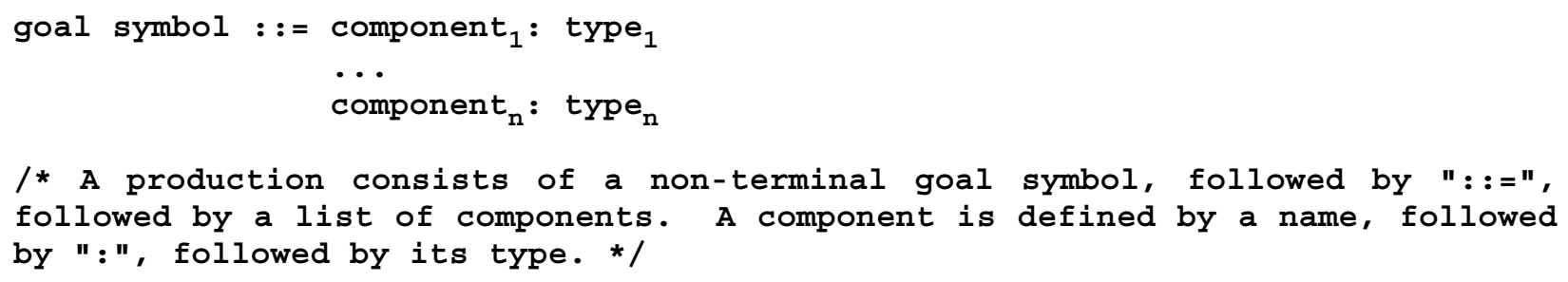

Figure 3-1: Production

Action equations are associated with particular productions in the syntax description in the same manner as the semantic equations of attribute grammars. The productions define the composition of the non-terminal nodes in the syntax tree representing the program. Figure 3-1 illustrates the context-free grammar notation adopted for action equations. This notation is based on the Interface Description Language [44,58] (IDL) developed as part of the Ada implementation effort, and has been used previously in DOSE [39], an interpretive LBE generation system. Only the abstract syntax is shown; the concrete syntax, or 'syntactic sugar', is omitted throughout this article. This syntax description notation is not in any way integral to action equations, and any other context-free grammar notation could be substituted - the only difficulty might be a less readable semantics description.

A non-terminal goal symbol is associated with a list of components, where each component has a name and a type. The same symbol may appear as the goal of multiple productions, indicating several alternative derivations; for example, a STATEMENT may be an if statment, a while statement, a compound statement, etc. The type of a component may be a non-terminal symbol, a terminal symbol or a sequence. Terminal symbols correspond to the primitive types of conventional programming languages. The set of terminal symbols available is specific to the implementation of the environment generation system, but would typically include identifier, integer, real, boolean, string and text. The sequence constructor is in contrast to the tail recursive method of defining lists using non-terminal, terminal and empty symbols. In each case, the sequence definition includes the element type.

In addition to alternative sets of components, a group of attributes and events may be 


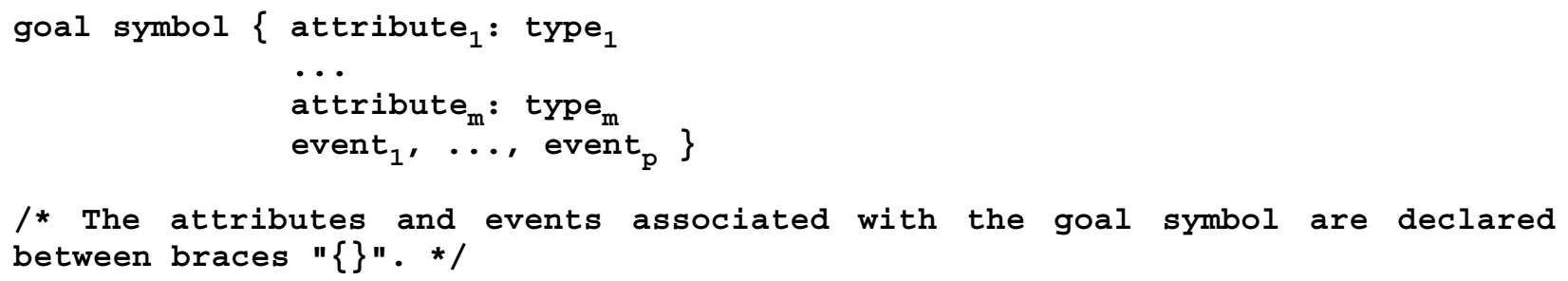

Figure 3-2: Attribute and Event Declarations

associated with each goal symbol as depicted in figure 3-2. Each node defined by this symbol is decorated with this set of attributes, which represent the current values of its properties; the events are attached to action equations that manipulate these properties. Attributes are typed in the same manner as components, where the type is given as a non-terminal symbol, a terminal symbol or a sequence.

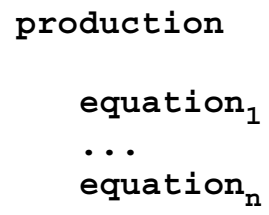

Figure 3-3: Action Equations

The action equations associated with a particular production describe the semantics processing for each node that is an instance of the production. A production and its action equations are depicted in figure 3-3. As in attribute grammars, the order equation ${ }_{1}, \ldots$, equation ${ }_{n}$ shown does not imply any sequencing among these equations, or that they should be evaluated in this or any other particular order.

location := function(attributes and terminals)

Figure 3-4: Assignment/Constraint Equation

There are five kinds of action equations: assignments, conditionals, constraints, delays and propagates. The assignment and constraint equations both have the form shown in figure 3-4, and the distinction is due to whether or not the equation is attached to an event. Assignments are, by definition, attached to events while constraints, by definition, are not; this is explained in the next section. For both, the right hand side denotes some function of attribute values and terminal 
node values; these values are called the arguments or inputs of the equation. The value computed by this function, called the result or output, is placed in the location given on the left hand side of the equation. The location is typically the name of an attribute, in which case the equation is identical in form to the semantic equation of attribute grammars.

The location may also be given as an address expression applied to an attribute name. This permits the modification of a previously calculated attribute value. This divergence from the attribute grammar paradigm has a significant implication: Attribute modification, together with events, make it possible for an attribute to reflect the history of program modification and/or execution. Otherwise, each attribute would of necessity be derived solely from the program text as explained previously.

The third alternative is for the location to be an address expression applied to a node in the syntax tree. Thus, the equation directly modifies the program as seen by the user, which is not possible in the pure attribute grammar paradigm. It might be argued that modification of the program by the environment should never be possible, on the grounds of the "principle of least astonishment'. This argument assumes the programmer does not expect the programming environment to change his program, but exactly the opposite is true in transformational programming environments, formal $[4,19,47,57]$ or informal [62]. There is no reason the programmer should expect less from an LBE; in particular, manipulation of the program text by action equations is one mechanism for implementing transformations.

If expression

Then equation (s)

Else equation(s)

Figure 3-5: Conditional Equation

The conditional equation consists of an expression and two sets of equations, as depicted in figure 3-5. The conditional equation specifies that when the expression is true, the first set of equations must hold; when the expression is false, the (optional) second set is applicable. All conditional equations can be expressed instead by permitting conditional expressions within other kinds of equations, and are thus only convenient syntactic sugar that provide no new meaning. 


\subsection{Events}

The propagate and delay equations, as well as the distinction between assignments and constraints, requires a discussion of events. Events correspond roughly to user commands. There are two kinds of events, standard events and implementor-defined events. Each standard event coincides with a primitive operation accessible to the user, including at least:

Create $\quad$ Replace the current placeholder with a newly created instance of a specified language construct.

Delete $\quad$ Remove the subtree rooted at the current node from the syntax tree and replace it with a placeholder.

Clip Save a copy of the current subtree in a register.

Insert Replace the current placeholder with a copy of a previously clipped subtree.

Enter Move the editing cursor to a specified child of the current node.

Exit Move the editing cursor to the parent of the current node.

The same set of standard events may appear in the semantic description of every environment just as the same set of standard commands are available in every environment. In contrast, an implementor-defined event is an identifier introduced by the implementor for a particular environment. An implementor-defined event corresponds to a user command available only in the specific environment, such as Execute or CrossReference a Pascal program.

production

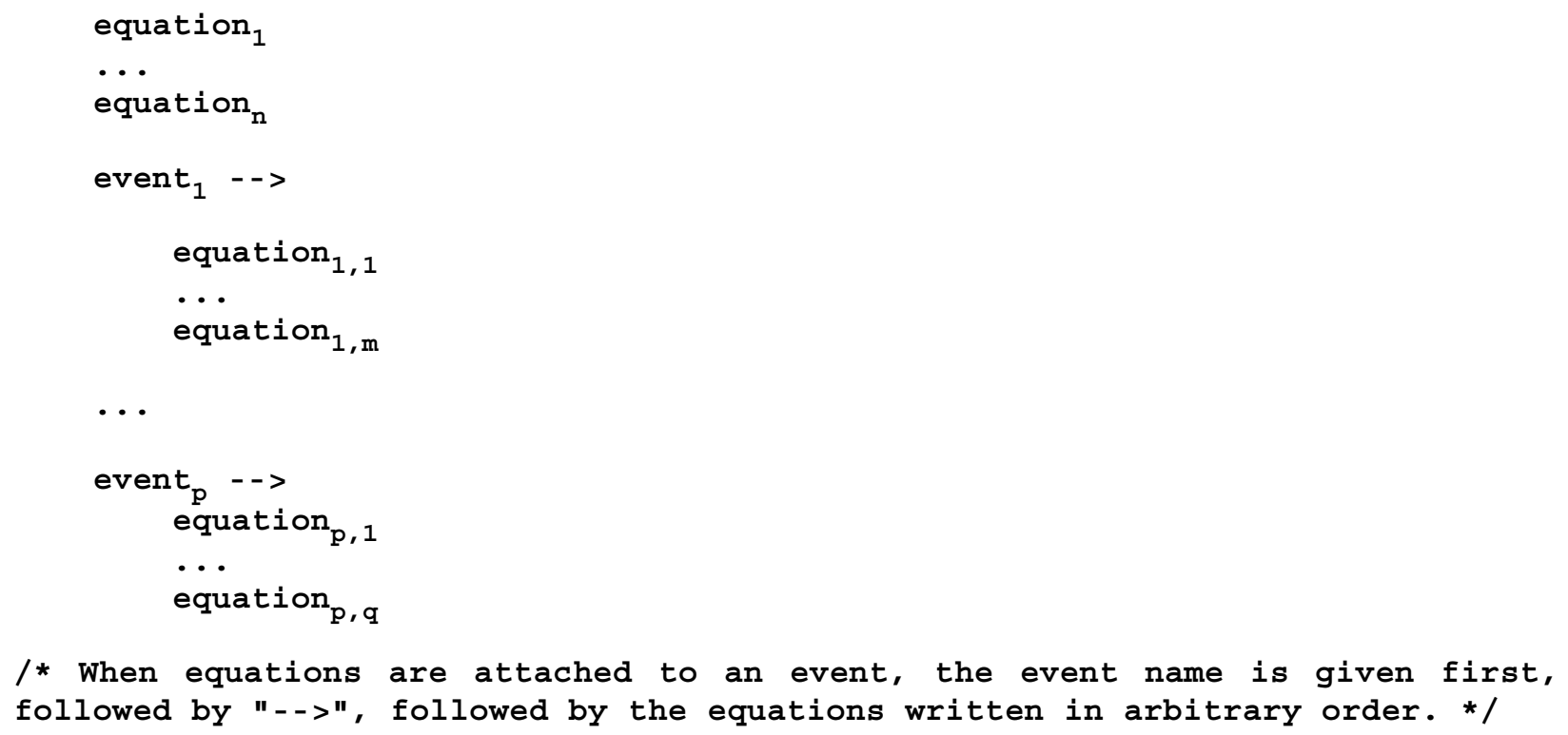

Figure 3-6: Attaching Equations to Events 
Each action equation associated with a particular production may or may not be attached to a particular event. Some action equations associated with a particular production may be attached to one particular event, other action equations associated with the same production may be attached to a different event, and still other equations may not be attached to any event at all. Figure 3-6 illustrates a number of equations associated with the same production: equation ${ }_{1}$ through equation $\mathrm{n}$ are not attached to any event, equation $\mathrm{n}_{1,1}$ through equation $_{1, \mathrm{~m}}$ are attached to event $_{1}$, and so on, through equation $\mathrm{p}_{\mathrm{p}, 1}$ through equation $_{\mathrm{p}, \mathrm{q}}$ attached to event $_{\mathrm{p}}$.

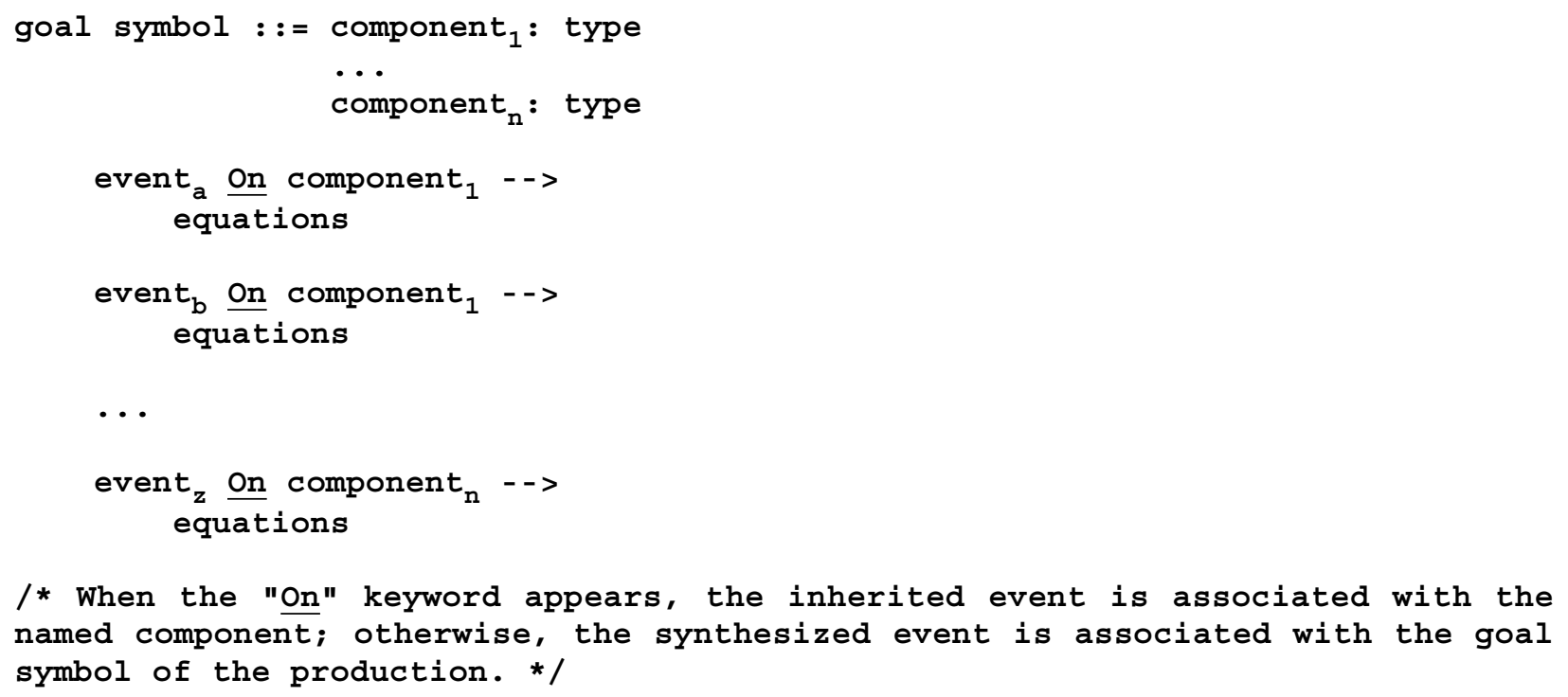

Figure 3-7: Inherited Events and Equations

The semantic equations of attribute grammars may define the value of an attribute associated either with a component of the production (the corresponding attribute is inherited) or with its goal symbol (the attribute is synthesized). Events are similarly inherited or synthesized. The events shown in figure 3-6 are associated with the goal of the production, and thus synthesized. An inherited event, with its attached equations, is associated with a component name as shown in figure 3-7. In this case, the event name must be declared for each goal symbol that is a legal type for the component. Unlike the attributes of attribute grammars, the same event may appear in both productions defining a node and thus may be both inherited and synthesized. Further, the same event may be inherited multiply with respect to the same production, due to multiple associations of the same event with incidentally the same element of a sequence (for example, "event ${ }_{\mathrm{a}}$ On component $_{\mathrm{n}}[\mathrm{i}]$-->" and "event ${ }_{\mathrm{a}}$ On $_{\text {component }}[\mathrm{j}]-->$ " where the ith element is also 
the jth). The multiple sets of equations attached to the event are concatenated, as if they had not been associated with different productions and/or different component descriptions.

Action equations that are not attached to a particular event fill the same role as the semantic equations of attribute grammars in the sense that they may be reevaluated when the program changes. It does not matter which particular user command caused the program modification, since all are treated as subtree replacements. These equations are said to be permanently active. In contrast, the collection of action equations attached to a particular event are active only when the event is explicitly selected by a user command or explicitly propagated by a propagate equation, explained shortly. These equations are passive at all other times. Only active equations may be evaluated, and an equation activiated by an event immediately becomes passive again after its evaluation. The collection of action equations attached to an event describe the semantics processing, or tool operation, for the user command that corresponds to the event.

An assignment equation is attached to an event. When activated by selection of the event, it computes the value denoted by its right hand side and assigns this output to the location on its left hand side. A constraint equation cannot be attached to an event. Whenever an input to its right hand side changes in value, it updates the location on its left hand side to maintain the equality. The distinction is necessary because constraints must always hold, as invariants, while assignments are evaluated exactly once when activated. Constraints are typically reevaluated in response to subtree replacements, but may also be reevaluated when an assignment changes the value of an input to a constraint.

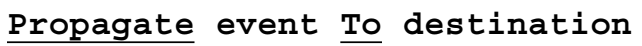

Figure 3-8: Propagate Equation

The user explicitly selects an event by moving the editing cursor to the node and entering the command corresponding to the desired event. Propagation of events from one node to another is done with a propagate equation, as depicted in figure 3-8. When activated, the equation propagates the given event to the indicated destination node. This has the effect of activating certain equations associated with the production that defines the destination node, in particular, all those equations attached to the named event; if this set is empty, then no new equations are 
activated. As with the arguments of semantic equations in attribute grammars, the destination is normally restricted to the children, siblings, parent and other ancestors (reached through uplevel addressing [42]). However, it is also possible to propagate from an identifier definition to its use(s) or from a use to its definition(s), as described in the next section.

Delay Until event $\underline{\text { At }}$ receiver

Figure 3-9: Delay Equation

The final kind of action equation is the delay equation, which has the form shown in figure 3-9. When activated, a delay equation suspends all currently active equations until the named event is selected for the indicated receiver node. Like the destination node of the propagate equation, the receiver node is restricted to the parent, ancestors, siblings and children of the current node. When the event is selected with respect to the receiver node, the previously suspended equations are reactivated. The event may be selected either by a user command or by a propagate equation resulting from a user command. In the latter case, the previously suspended and now activated equations are in addition to any equations that may be active at the time of the event. The receiver node of a delay equation is optional; if omitted, then the delay equation refers to the selection of the named event when the editing cursor is at any node.

When a group of equations are attached to the same event, both as synthesized and inherited, there is a specific ordering among the different kinds of equations. In particular, any delay equations are evaluated first and, in effect, simultaneously. Thus all other equations attached to the same event are suspended by the delay equation(s); if there are multiple delays, then all the named events must be selected for their receivers to reactivate the suspended equations. If there are no delay equations, then any assignments and conditionals attached to the event are evaluated in any order (except as noted below) consistent with the dependencies among inputs and outputs of the assignments and the inputs of the expression parts of the conditionals. Any constraints, and conditionals not attached to the event, whose inputs are among the outputs of these assignments are also evaluated if and only if the outputs are different than their previous values. Any propagate equations are evaluated last; any equations activated by these equations are, in effect, activated simultaneously.

This ordering among action equations is complicated by the conditional equation. Neither the 
then part nor the else part equations are themselves activated until after the expression has been evaluated, as soon as possible consistent with the partial ordering described above; an alternative semantics would evaluate these expressions as late as possible, but some such restriction is necessary to avoid non-deterministic behavior. After the value of the expression has been determined, then the appropriate set of equations are simultaneously activated, and the above rule applies regarding the previously active equations as well as the newly activated equations.

The main components of action equations paradigm have now been introduced. Section 4 describes the application of action equations to the description of programming language control constructs such as conditional statements, loops and procedure calls. Section 5 considers interactive execution of programs, including stream input/output and some typical features of symbolic debuggers. Section 6 discusses the translation and run-time support algorithms for generation of LBEs from action equations.

\section{Description of Control Structures}

\subsection{Flow of Control}

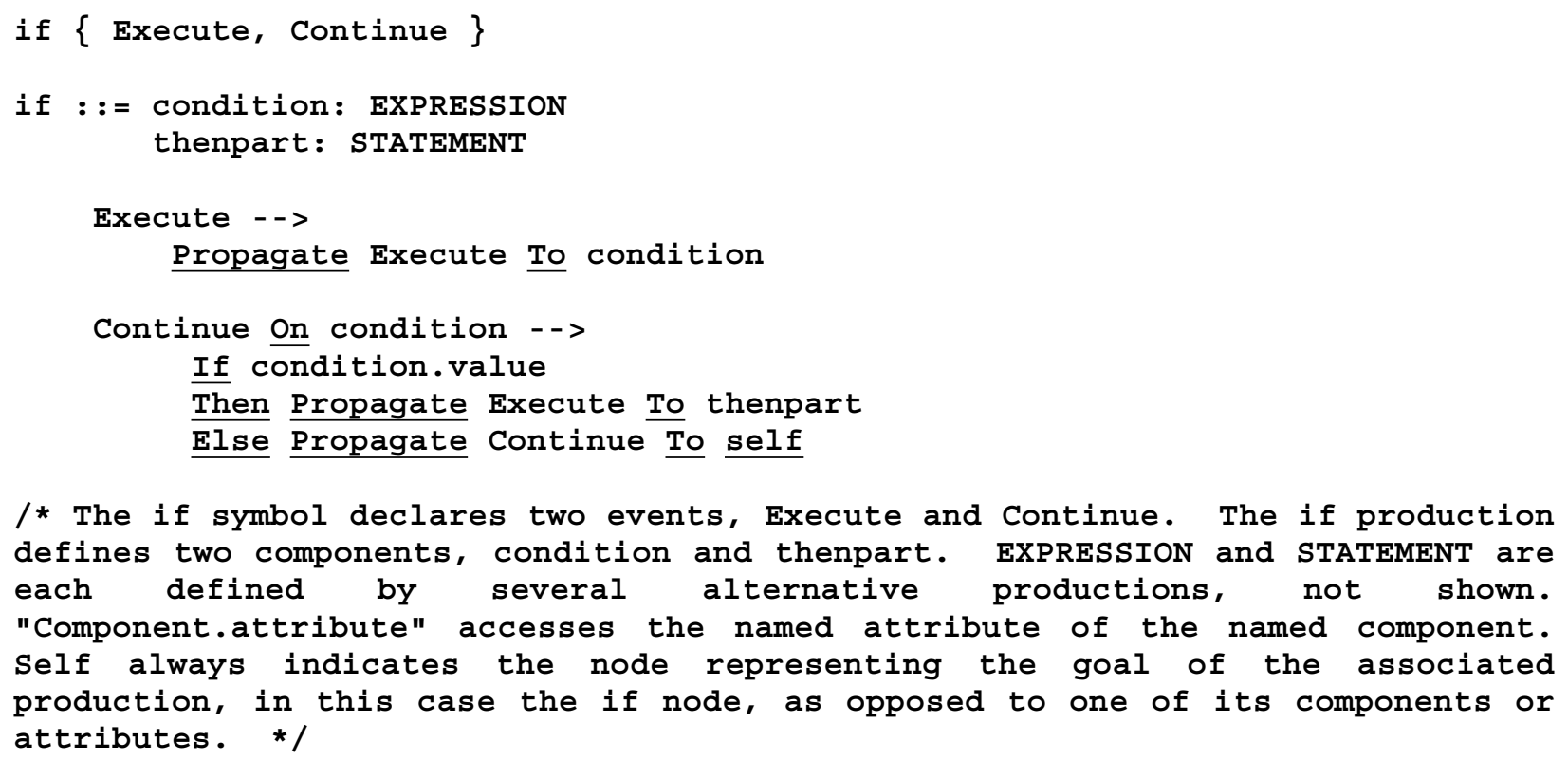

Figure 4-1: If Statement Syntax and Semantics

Figure 4-1 demonstrates the use of implementor-defined events and propagation of events in a simple description of interpretation. The implementor defines the Execute event to specify the 
execution of an if statement. When the Execute event is applied to an instance of the if production, the propagate equation selects the Execute event for the condition child of the if node. After any semantics processing involving the condition node are completed (including for example the setting of its value attribute), then the condition child propagates the Continue event to itself (the condition child). This Continue event activates the conditional equation. If the value of the value attribute is true, the Execute event is propagated to the thenpart child. If not, the if statement has completed execution, and the Continue event is propagated to itself (the if statement). Thus, the implementor-defined Continue event fills the role of the continuation of denotational semantics.

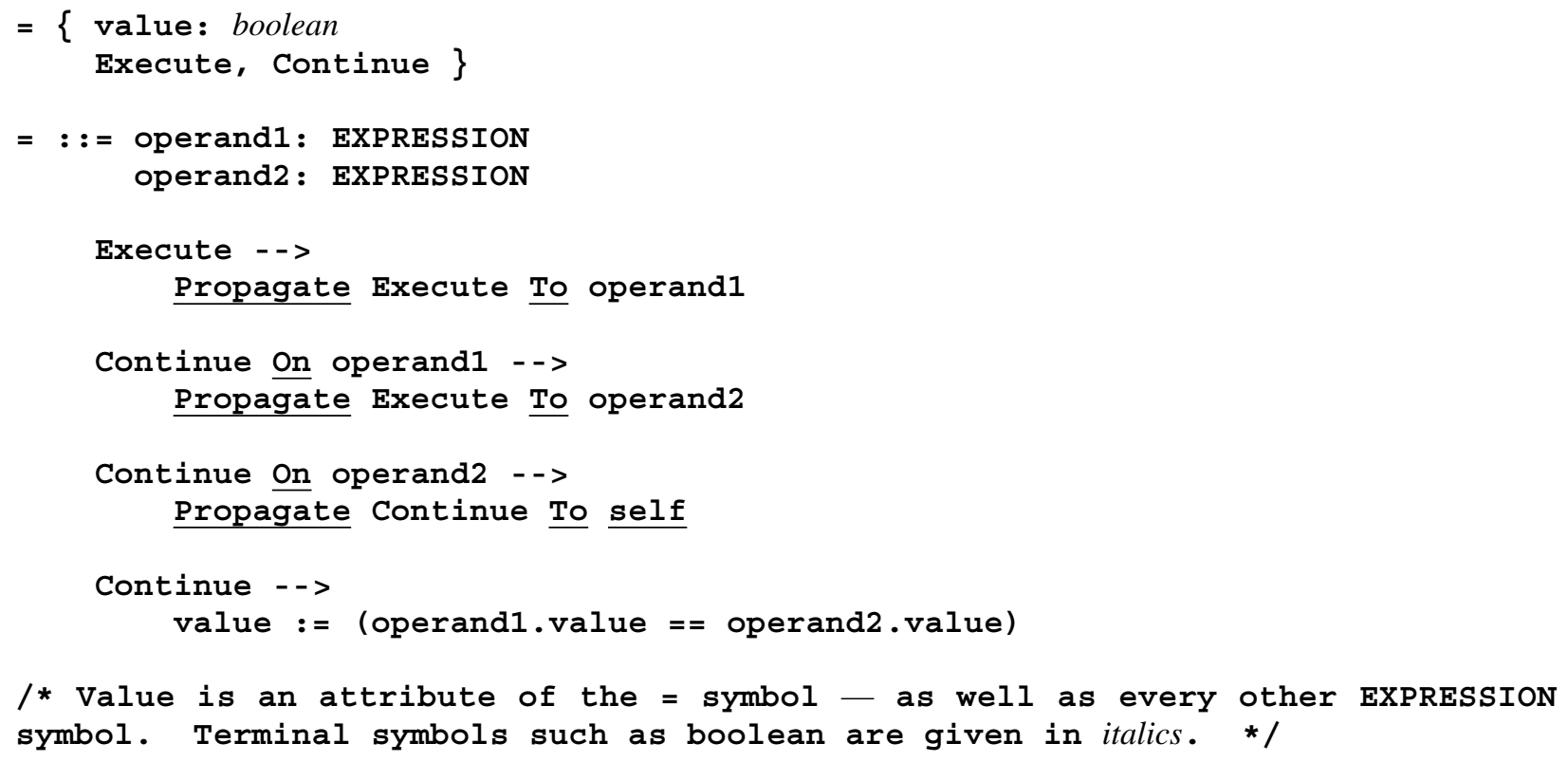

Figure 4-2: Action Equations for $=$ Production

Events and equations for one conditional expression, the = production, are shown in figure 4-2. When the Execute event is propagated to the = operator, the two operands are computed in order and then the value attribute of the = node is set to the result of comparing the two operands. Calculation of expression values does not, however, necessarily require this rather cumbersome action equations apparatus. Purely applicative expressions are handled in a natural way by pure attribute grammars, as demonstrated by Reps' and Teitelbaum's desk calculator [53], so this is not discussed further in this article. Expressions involving (potentially recursive) function calls and (multiple assignment) variables require a run-time stack, as discussed later in this section. 


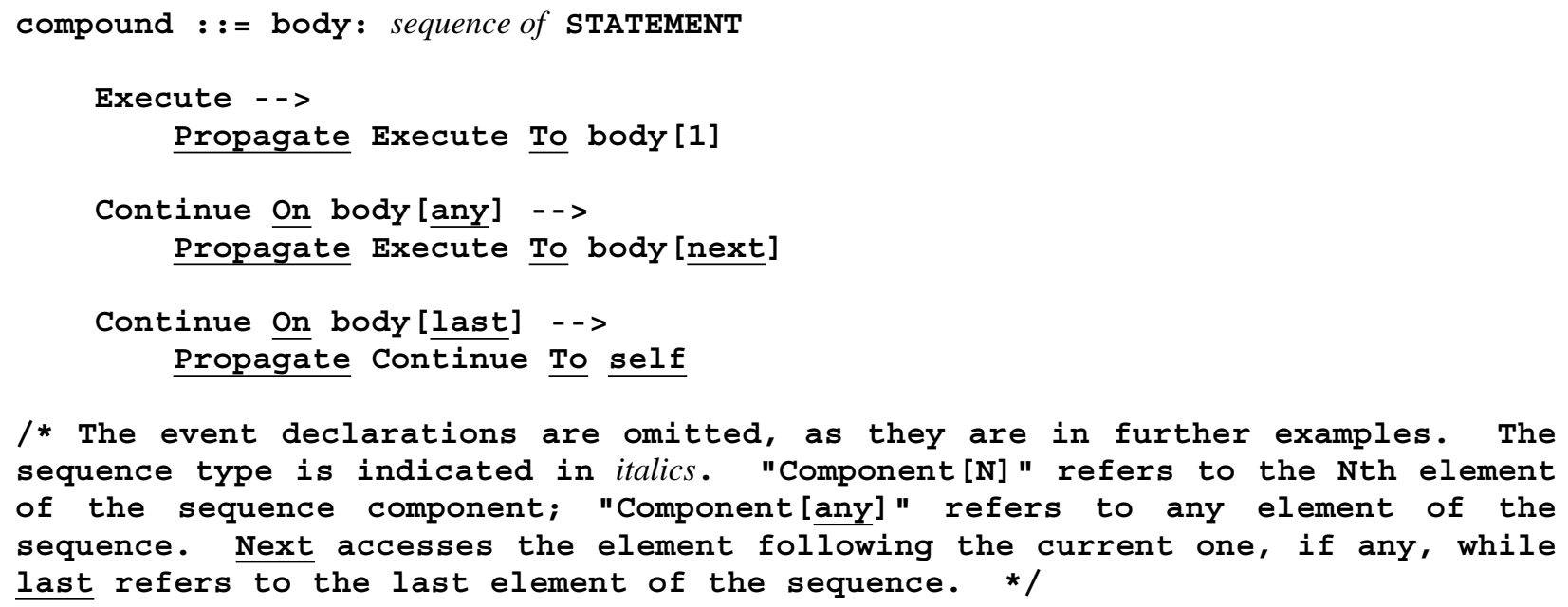

Figure 4-3: Compound Statement Syntax and Semantics

Figure 4-3 illustrates how event propagation works for a compound statement (i.e., a sequencer). The basic idea is that the Execute event propagates from the compound statement to the first statement in the body of the compound statement, from the first statement to the next statement in the body, etc. In the case of the last statement where multiple inherited events "Continue On body[any]" and "Continue On body[last]" both apply, both attached equations are executed. But "Propagate Execute To body[next]" has no effect since body[next] evaluates to $\underline{\text { nil. }}$

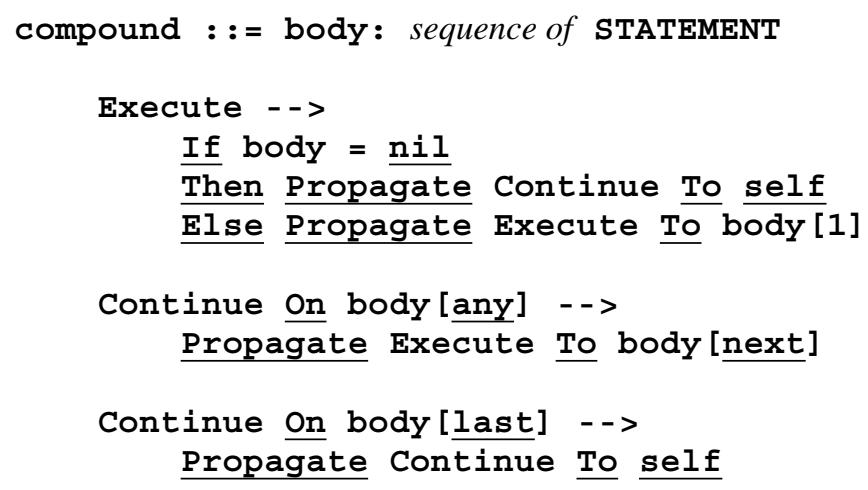

Figure 4-4: Compound Statement Syntax and Semantics, Revised

This discussion of the compound statement, and the previous example involving the conditional statement and = operator, have been simplified in that they do not consider the possibility that the body of the compound statement is empty, the condition and/or the thenpart 
of the conditional statement is missing, or one or both operands of the $=$ operator are meerly placeholders, respectively. The analogous issue arises in the semantic equations of attribute grammars, and is solved there by requiring the implementor to provide completing productions, which define the value of the attributes for every potential placeholder. Action equations also take this approach, and the implementor must explicitly treat the possibility of empty sequences and missing components. The compound statement example is revised accordingly in figure 4-4, where nil denotes an empty sequence; the rest of the examples in this article could be completed similarly, but this is not done to keep the examples simple.

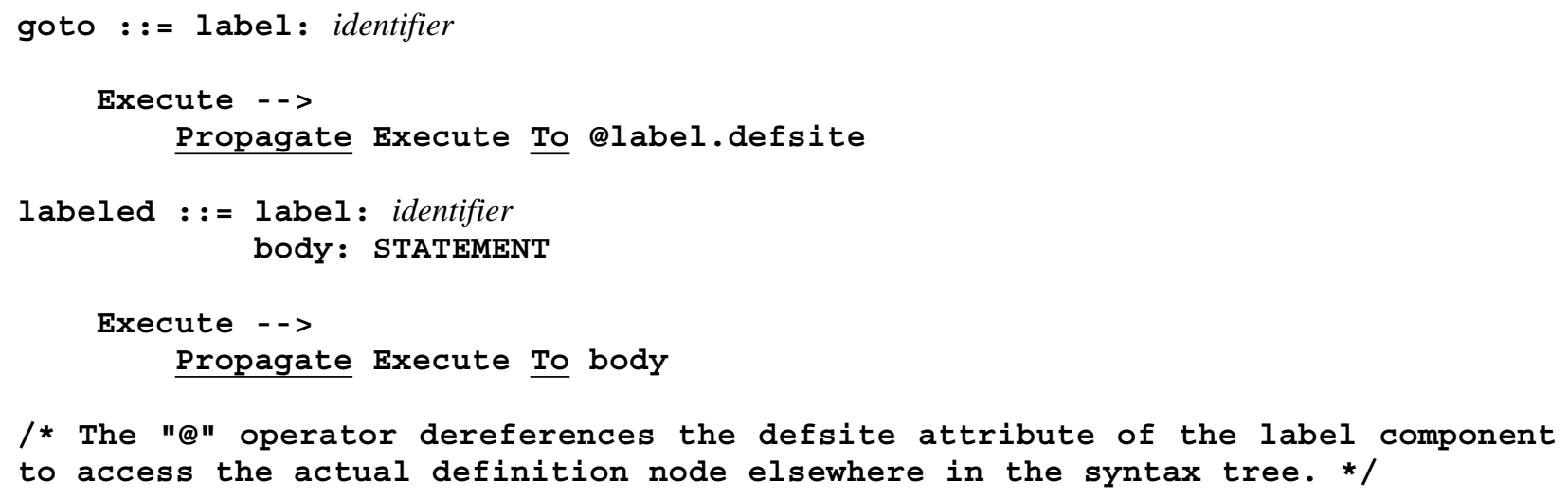

Figure 4-5: Goto Statement Syntax and Semantics

In order to describe the semantics of branch statements, some mechanism is needed to find the destination of the branch. This is done through identifier definition-use links. Several extensions to attribute grammars have been proposed $[9,27,28]$ that improve the efficiency of incremental attribute evaluation by linking the definitions and uses for each identifier. A change in an attribute value at a definition site is propagated along the links to dependent attributes at its use sites. Any one of these schemes can be used as the basis for propagating an event from the goto statement to the corresponding labeled statement as depicted in figure 4-5.

Figure 4-6 shows how the operation of a general loop statement is described using action equations. In this example, the initialization is performed first. Then the condition is tested. If true, the body of the loop is executed. Now reinitialization, condition testing and the body are repeated until the condition becomes false.

Notice that the propagate equations in this example denote a circular dependency. The 


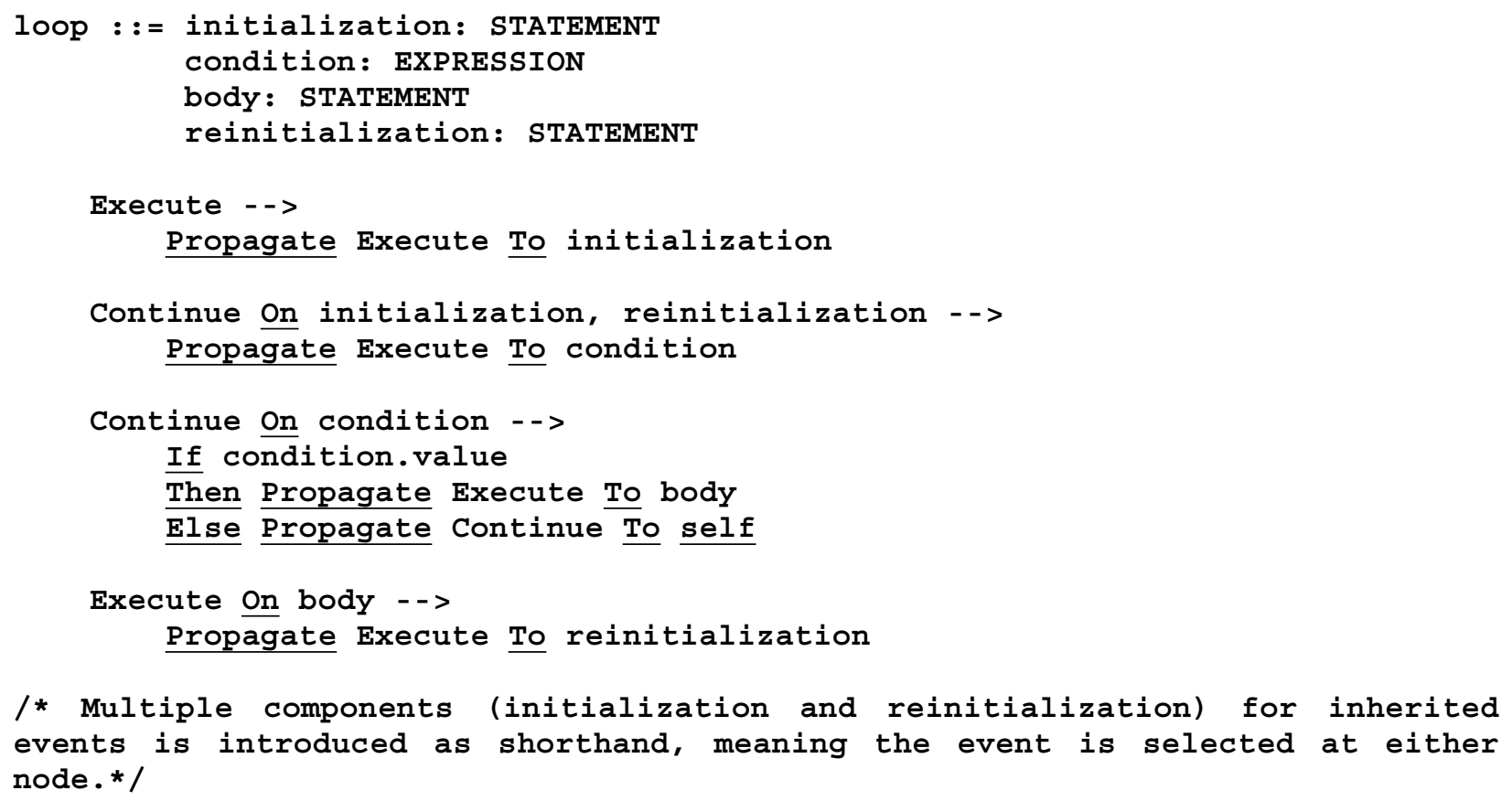

Figure 4-6: Loop Statement Syntax and Semantics

condition propagates to the body, the body propagates to the reinitialization and the reinitialization propagates to the condition. Although circular attribute grammars are problematical for non-incremental evaluation [15] and rarely handled by incremental evaluators (work by Walz and Johnson is a notable exception [61]), circularities among propagate equations pose no difficulties. If the user of a generated environment writes an infinite loop, then the propagation never terminates, to preserve correct dynamic semantics processing; if the loop does terminate, then the propagation terminates accordingly.

\subsection{Procedure Call and Return}

A likely syntax description for a procedure definition, with its formal parameters and local variables, is shown in figure 4-7. The Execute event and attached equations for the procedure production are omitted, since they are essentially identical to those for the compound statement. The procedure symbol has an AR attribute that acts as a template for the procedure's activation record during execution. Frame is a non-terminal symbol, where the details of any particular frame node are computed by constraints just as is done by attribute grammars. For example, the size (in bits, bytes or words) of each formal and local might be computed from its type and then its offset within the activation record determined by the cumulative size (and required 


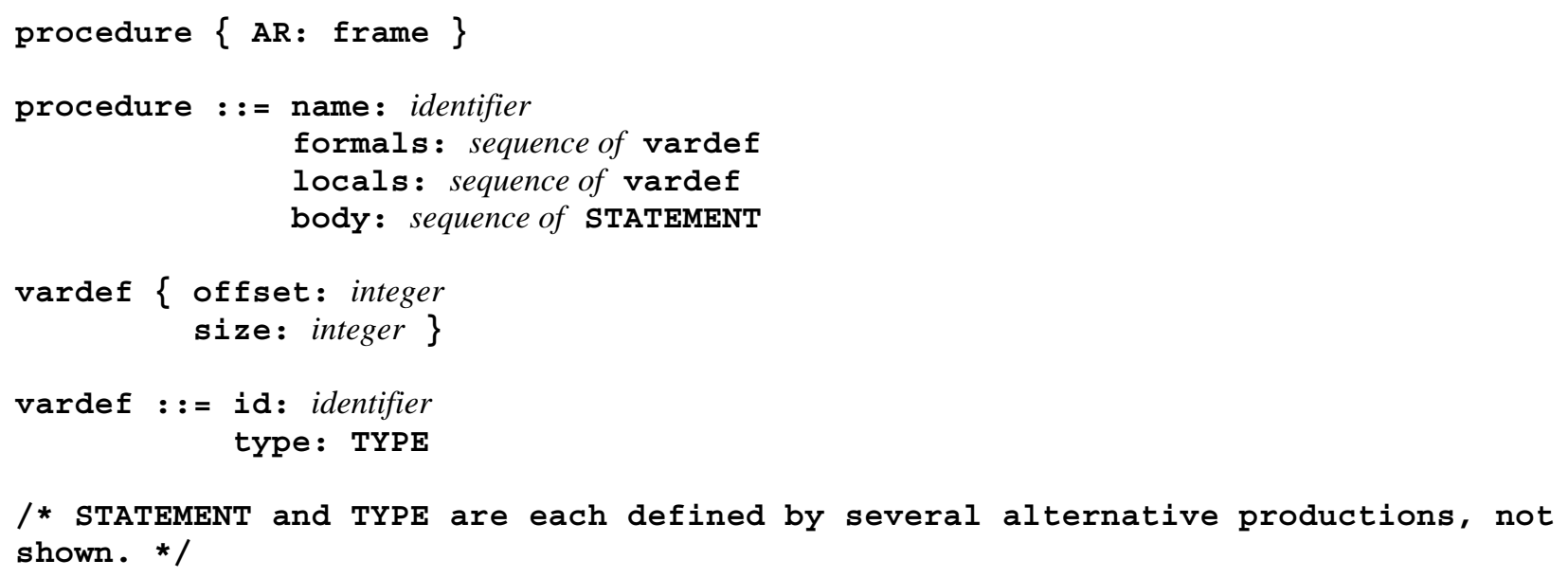

Figure 4-7: Procedure Definition Syntax

alignments). This would require each implementor to define a suitable representation for each datatype in his language [56]. One alternative would be to represent each data item as a node; this is much less efficient at execution time but much more expedient at environment description time.

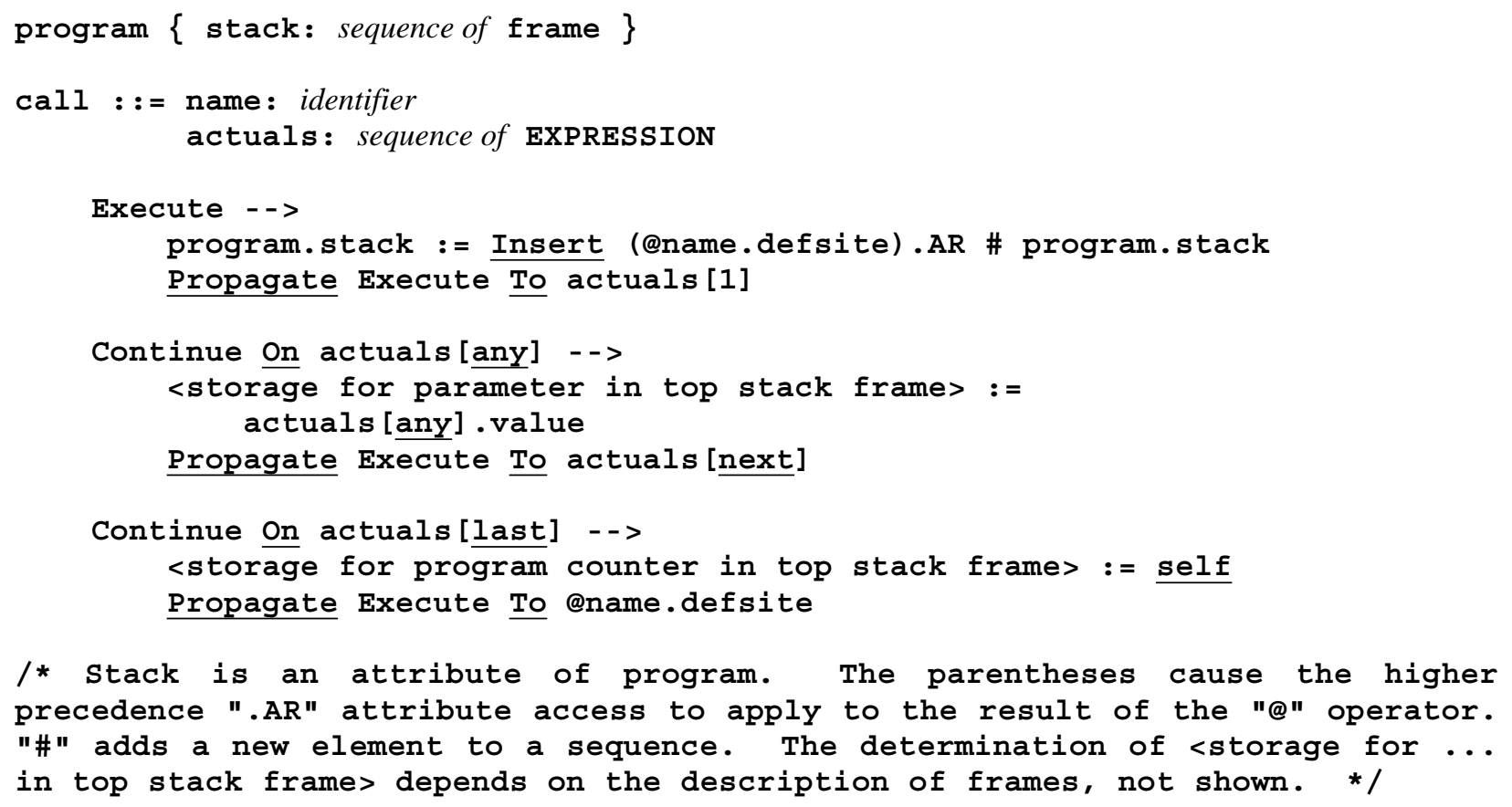

Figure 4-8: Call Statement Syntax and Semantics

The syntax and semantics of the procedure call statement are given in figure 4-8. The run-time 
stack is represented as a sequence of frame nodes maintained as an attribute of the program node. Execution begins by applying the internal version of the user-level Insert command to insert a copy of the procedure's activation record at the top of the stack. The actual parameter expressions are then executed, and their resulting values stored in the corresponding slots in the activation record. After the last parameter is available, a reference to the call statement is saved as the program counter and then execution propagates to the procedure definition.

Remember that the apparent circularity of program.stack on both sides of the equation is not a problem, since it occurs in an assignment rather than a constraint. Such 'circularities' are necessary for maintaining history information, where the new value of an attribute is computed by directly modifying its old value. Potential circular dependencies among constraints are handled as in attribute grammars, by separating into in and out attributes where the synthesized out attribute is the appropriate function of the inherited in attribute.

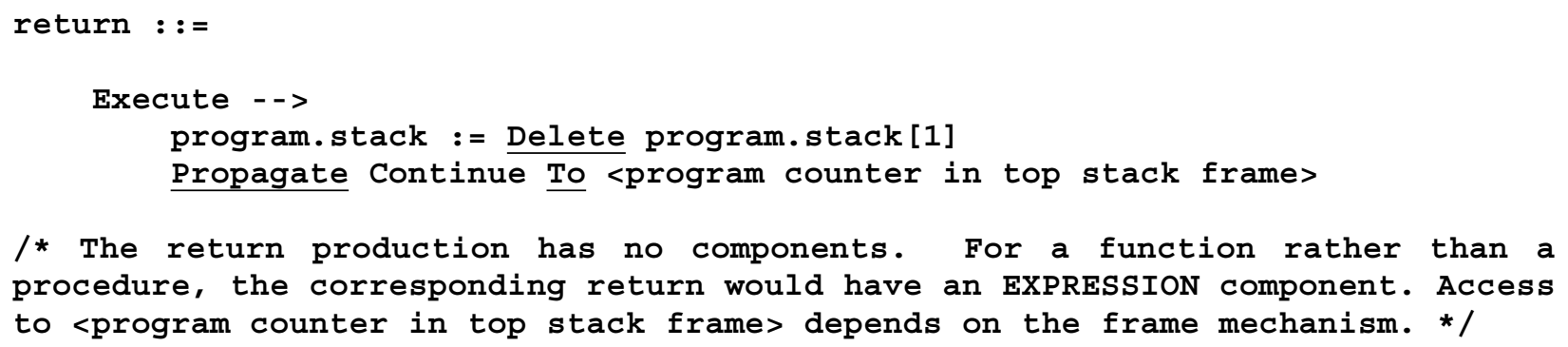

Figure 4-9: Return Statement Syntax and Semantics

Figure 4-9 gives the equations for execution of a return statement. The top stack frame is removed from the stack using the Delete command, and the continuation propagates to the original call statement.

\section{Interactive Execution and Debugging}

\subsection{User Input/Output}

Figure 5-1 illustrates one mechanism for representing sequential I/O, for either the terminal display and keyboard or ASCII files. For simplicity, each channel consists of both an input stream and an output stream, where each stream is a sequence of buffered text lines. Standard input and standard output are combined in the first channel. 


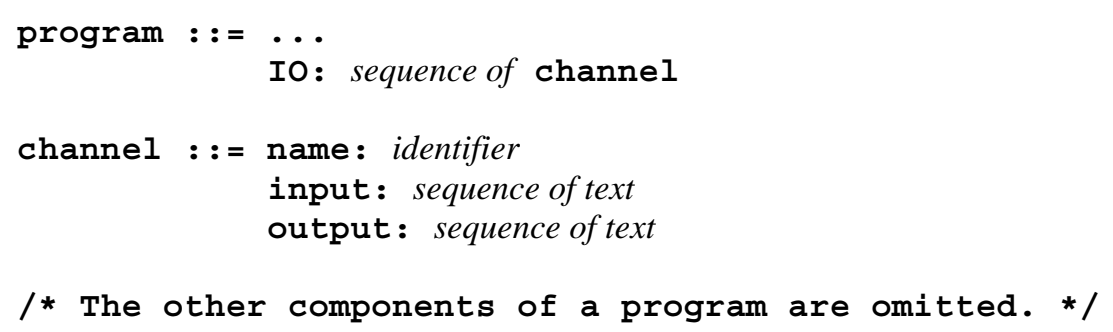

Figure 5-1: Input and Output Streams

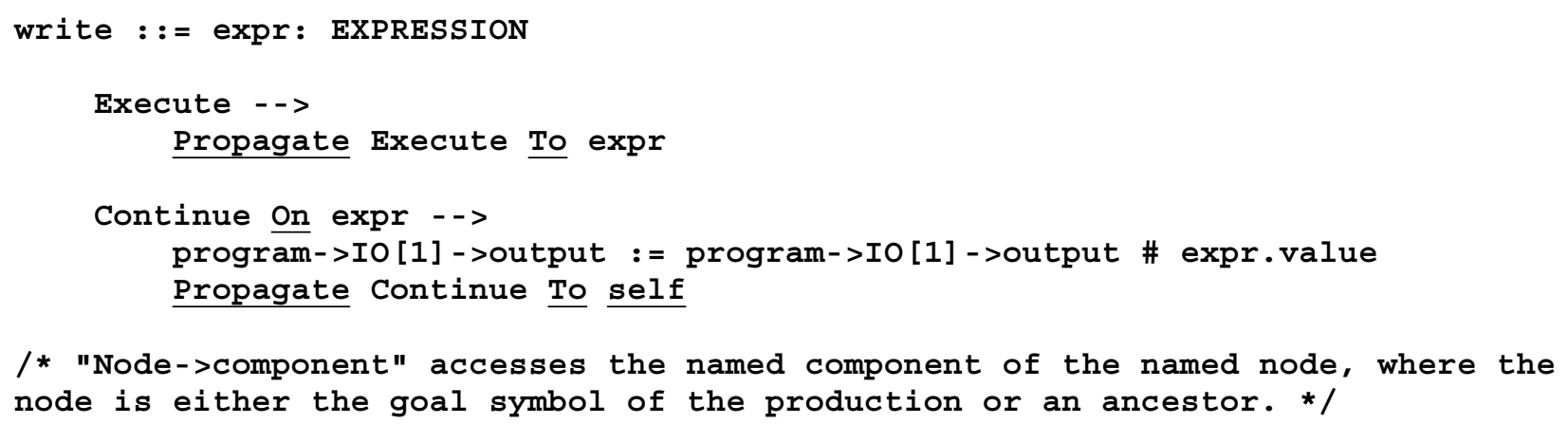

Figure 5-2: Write Statement Syntax and Semantics

Figure 5-2 gives the syntax and semantics of a simple write statement. When the Execute event is applied to an instance of the write production, the Execute event is propagated to compute the value of the expression. On the continuation, the text representation of this value (as determined by the implementation of the underlying environment generation system) is concatenated to the end of the output stream. The output stream is automatically redisplayed on the screen after every update. Various kinds of unparse schemes have been proposed for defining the concrete syntax necessary for displaying the program [24, 29, 53] or distinct views of the program $[21,50]$. The action equations paradigm assumes the availability of one of these mechanisms for display purposes.

The read statement is slightly more difficult and requires a delay equation. The first equation attached to the Execute event for the read production, given in figure 5-3, requests the user to select the Create event to add a new last element to the input stream. The delay equation has the effect of suspending program execution until the user has entered a new line of input by appending to the sequence of text lines that represents the standard input. Only then is the last (new) element of the input sequence stored as the value of the variable given in the read 


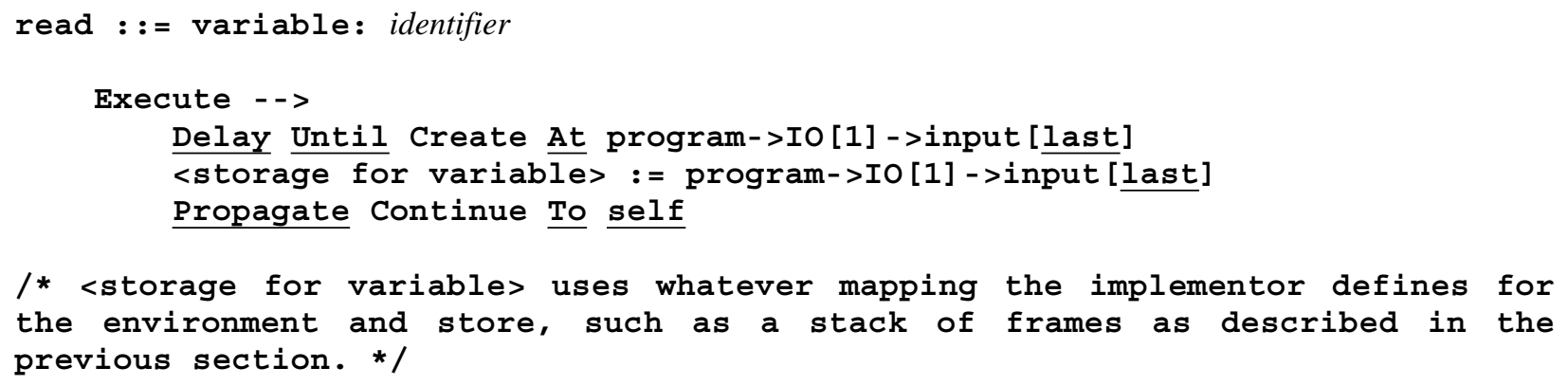

Figure 5-3: Read Statement Syntax and Semantics

statement.

\subsection{Program Suspension and Continuation}

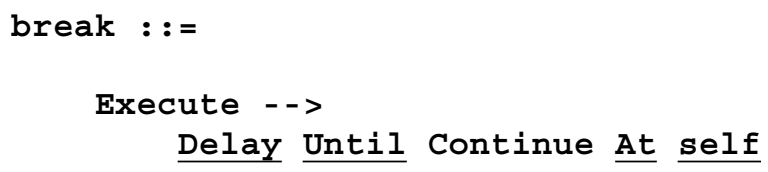

Figure 5-4: Break Statement Syntax and Semantics

The delay equation is also instrumental in specifying debugging facilities such as breakpoints and singlestepping. Figure 5-4 shows hows a breakpoint might be described. This example follows the precedent set by Feiler in his thesis [17] (and elsewhere [16]) as to how the user specifies a breakpoint before or after a particular statement. It assumes that the programming language has been extended by a special break statement. The user designates a breakpoint by inserting a break statement at the desired position in the program text. The interpreter suspends program execution when the Execute event is propagated to the break node, presumably by an equation for some other node.

The user continues from a breakpoint by entering the Continue command when the editing cursor is pointing to the break statement. Selecting the Continue event at some other position in the program would activate the equations attached to the Continue event for the corresponding production, effectively starting up a separate execution thread at that position. A conditional breakpoint might be defined by adding an expression to the break statement and enclosing the delay equation inside a conditional equation. 


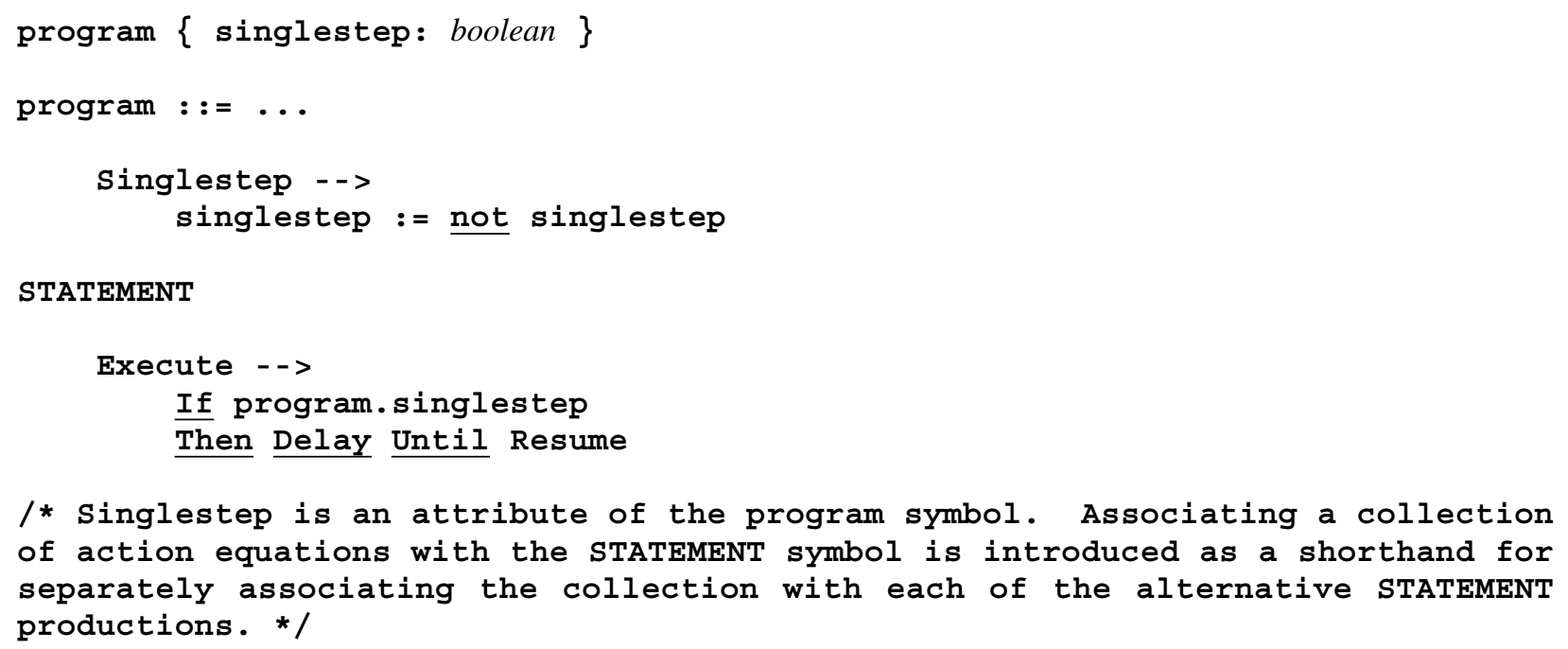

Figure 5-5: SingleStepping

The description of singlestepping is similar. Figure 5-5 depicts Singlestep as an implementordefined event that toggles singlestepping on and off, by changing the value of the singlestep attribute. The delay equation is associated with every STATEMENT production. If singlestep mode is on, then the interpreter suspends before the execution of each statement, until the Resume event. Since no receiver is specified in the delay equation, it does not matter where the editing cursor is when the user enters the Resume command. When the user selects the Resume event, the interpreter awakens and continues execution with the current statement.

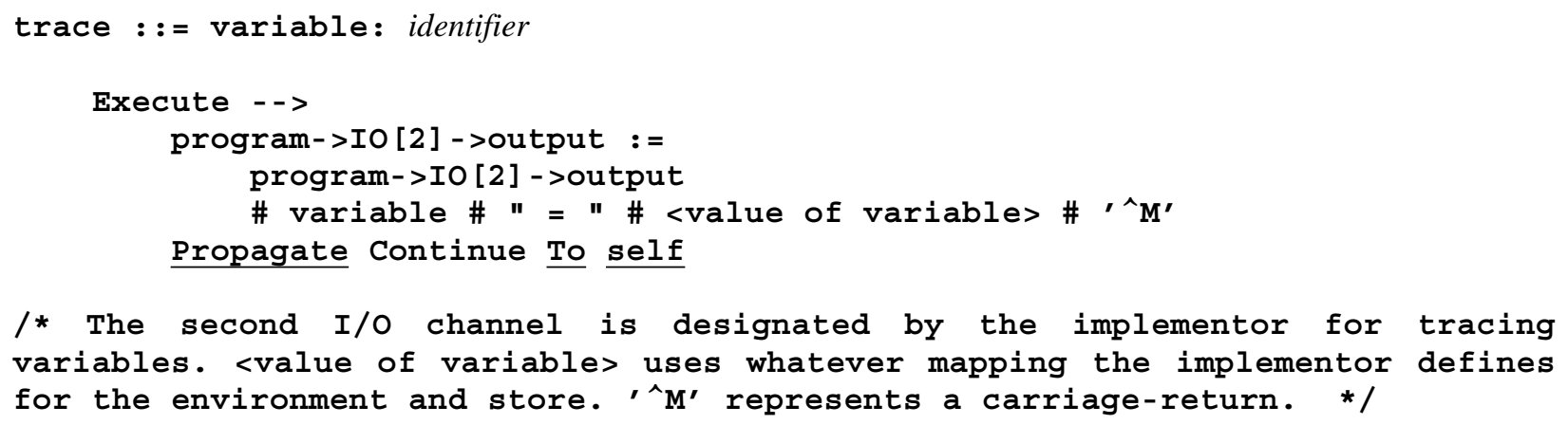

Figure 5-6: Trace Statement Syntax and Semantics

Tracing is another debugging facility that can be described by extending the target programming language with a special statement. As illustrated in figure 5-6, a variable might be traced by inserting a trace statement with the variable name at every point where display of the 
variable's value is desired. The trace statement is executed similarly to the write statement: the variable's current value is appended to the output stream of a designated I/O channel and displayed using the standard unparse mechanism. Alternatively, the explicit trace statement can be avoided by attaching a conditional equation to the Execute event for the assignment statement, to perform the trace when any variable in some list (given by an input channel) is assigned.

\section{Implementation Algorithms}

The implementation of action equations consists of two parts, translation and run-time support. Both parts involve an adaptation of the Reps, Teitelbaum and Demers algorithms [52] for generation of LBEs from attribute grammars. Reps' algorithms work roughly as follows. The translator takes as input the environment description and produces as output (1) various tables reflecting the syntax description; (2) a local dependency graph for each production representing the dependencies among the attributes that appear in its semantic equations; and (3) a procedure for each semantic equation, which carries out the actual evaluation of the equation.

After each subtree replacement, Reps' run-time support constructs a scheduling graph by grafting together two projections of the local dependency graph for the root of the replacement subtree, one denoting the transitive dependencies among the attributes of the node via its parent and siblings, and the other the transitive dependencies among the attributes of the node via the subtree. The attributes represented in the scheduling graph are reevaluated in the order given by a topological sort of the graph. The attributes represented by independent vertices (i.e., those vertices with no incoming edges) are reevaluated first.

If the execution of a semantic equation results in a value different from the previous value of the attribute, then the scheduling graph is expanded to include the projected local dependency graphs for all attributes that depend directly on the changed attribute. The expansion involves adding edges representing transitive dependencies for all of these attributes that were not

previously part of the scheduling graph. Whether or not the attribute changed in value, it and all its outgoing edges are now removed from the graph and evaluation continues with those attributes now represented by independent vertices. This process continues until the scheduling graph becomes empty, which is guaranteed to happen eventually if the attribute grammar is noncircular. (Algorithms to detect circularity in an attribute grammar are exponential [30], so 
whether or not a given attribute grammar is non-circular is often determined by inspection.) This evaluation algorithm is asymptotically optimal in the sense that the number of attribute evaluations is proportional to the number of attributes that are necessarily reevaluated. (The efficiency may be improved by maintaining additional data structures, making it possible to avoid all unnecessary evaluations.)

The adapted set of algorithms operate as follows. During translation of action equations, syntax tables and procedures are generated similarly to attribute grammars. The important distinction is that a local dependency graph is constructed for each event, whether synthesized or inherited, associated with each production. The graph represents the dependencies among the equations attached to the event rather than the attributes that appear in these equations; this is necessary because the outputs of action equations may be placed in locations within an attribute or within the syntax tree and these locations may be computed during action equation evaluation. In each graph, there are no incoming edges for each delay equation (to ensure that they are evaluated first), an outgoing edge from every delay equation to every other kind of equation, and an incoming edge from every other kind of equation to every propagate equation. There is also a local dependency graph for the set of equations - constraints and conditionals - not attached to any event.

After each subtree replacement, a scheduling graph is constructed from the projected local dependency graphs for the equations not attached to any event and also the two local dependency graphs (synthesized and inherited) for the equations attached to the standard event corresponding to the user command that caused the subtree replacement. In response to each user command corresponding to a cursor movement or an implementor-defined event, a scheduling graph is constructed from the two local dependency graphs for the equations attached to that event. In either case, the run-time support then follows the topological sort/graph expansion process described above.

As explained previously, the evaluation of several delay equations is treated as simultaneous and results in saving the scheduling graph together with a representation of the required event/receiver pairs. Once the full set of events has been selected, in any order and spread out over any period of time, the saved scheduling graph is grafted together with the then current scheduling graph. The evaluation of several propagate equations is also treated as simultaneous, and results in a new scheduling graph (by definition, the previous graph is empty except for the 
propagate equations), which includes the two local dependency graphs for all the propagated event/destination pairs.

The incremental action equation evaluation algorithm is asymptotically optimal in the same sense as the base incremental attribute grammar evaluation algorithm. In the case of a subtree replacement, constraints are treated as if they were semantic equations and evaluated via the identical mechanism. In the case of equations attached to an event, each equation is evaluated exactly once for each selection of the event as required by the semantics of action equations, and the number of constraint evaluations is proportional to the number of constraints that must be reevaluated.

\section{Conclusions}

The purpose of this article is to demonstrate that attribute grammars can be easily extended to specifying dynamic semantics in addition to static semantics. The action equations paradigm does this by making the attribute grammar itself dynamic, where some semantic equations are active and others are passive. Equations are changed from passive to active to passive again according to external user commands and internal computations involving the propagate and delay equations. Action equations also augment attribute grammars with limited side-effects, which make it possible to maintain the state of program execution and the history of user interactions with the environment.

This extension of attribute grammars was developed to permit generation of LBEs that support both static and dynamic semantics. Attribute grammars previously permitted generation of environments that support only static semantics. Action equations can also be applied outside LBEs to generate interpreters and debuggers, just as attribute grammars have been used to generate compilers.

\section{Acknowledgements}

The bulk of this research was conducted as part of my $\mathrm{PhD}$ thesis [36] at Carnegie Mellon University. I would like to thank my advisor, Nico Habermann, for his advice and support throughout my graduate student career. I would also like to thank the other members of my committee, Scott Fahlman, Elaine Kant and Bill Riddle, for their contributions. Discussions with David Garlan helped me work out the details of the action equations paradigm. I have more recently collaborated with David to develop Meld [37], an experimental language combining 
object-oriented and dataflow programming paradigms; Meld's syntax and semantics are loosely based on action equations and on the views David proposed in his thesis [22]. Simon Kaplan pointed out an error in one of the examples given in my dissertation, which is corrected here; Simon has also worked with me on parallel and distributed incremental evaluation algorithms for attribute grammars [38]. We have recently applied this work to a concurrent extension of action equations [40]. Finally, I would like to thank Dave Ackley, Nico Habermann, Josephine Micallef and the anonymous referees for their critical comments on earlier versions of this article; the comments of one of the referees were particularly useful and led to vast improvements in the form and content of the article.

A parallel/distributed implementation of Meld [41] has been completed by Nicholas Christopher, Seth Strumph and Shyhtsun (Felix) Wu under the direction of Wenwey Hseush and later Steve Popovich. Gaea, a rapid prototyping system for interpreter/debuggers based on the action equations paradigm, is currently being implemented by Travis Winfrey with the participation of Kok-Yung Tan, Matsuki Yoshino and Semyon Dukach. Both Meld and Gaea are being developed at Columbia University. Gail Kaiser is now supported by National Science Foundation grants CCR-8858029 and CCR-8802741, by grants from AT\&T, DEC, IBM, Siemens and Sun, by the Center of Advanced Technology and by the Center for Telecommunications Research.

\section{References}

u

1. Ambriola, Vincenzo, Kaiser, Gail E. and Ellison, Robert J. An action routine model for ALOE. Tech. Rept. CMU-CS-84-156, Carnegie Mellon University, Department of Computer Science, August, 1984.

2. Archer, James E. Jr. and Devlin, Michael T. Rational's experience using Ada for very large systems. First International Conference on Ada Programming Language Applications for the NASA Space Station, June, 1986, pp. B.2.5.1-B.2.5.11.

3. Bahlke, Rolf and Snelting, Gregor. "The PSG system: from formal language definitions to interactive programming environments". ACM Transactions on Programming Languages and Systems 8, 4 (October 1986), 547-576.

4. Balzer, Robert. "A 15 year perspective on automatic programming". IEEE Transactions on Software Engineering SE-11, 11 (November 1985), 1257-1268.

5. Barbuti, R., Bellia, M., Degano, P., Levi, G., Dameri, E., Simonelli, C. and Martelli, A. Programming environment generation based on denotational semantics. In Theory and Practice of Software Technology, North-Holland Pub. Co., New York, 1983. 
6. Bodwin, James, Bradley, Laurette, Kanda, Kohji, Litle, Diane and Pleban, Uwe. Experience with an experimental compiler generator based on denotational semantics. SIGPlan '82 Symposium on Compiler Construction, June, 1982, pp. 216-229.

7. Delisle, Norman M., Menicosy, David E. and Schwartz, Mayer D. Viewing a programming environment as a single tool. SIGSoft/SIGPlan Software Engineering Symposium on Practical Software Development Environments, April, 1984, pp. 49-56.

8. Demers, Alan, Reps, Thomas and Teitelbaum, Tim. Incremental evaluation for attribute grammars with applications to syntax-directed editors. 8th Annual ACM Symposium on Principles of Programming Languages, January, 1981, pp. 105-116.

9. Demers, Alan, Rogers, Anne and Zadeck, Frank Kenneth. Attribute propagation by message passing. SIGPlan '85 Symposium on Language Issues in Programming Environments, June, 1985, pp. 48-59.

10. Despeyroux, Thierry. Executable specification of static semantics. Semantics of Data Types International Symposium, New York, June, 1984, pp. 215-233.

11. Donzeau-Gouge, Veronique, Huet, Gerard, Kahn, Gilles, and Lang, Bernard. Programming environments based on structured editors: the Mentor experience. In Barstow, David R., Shrobe, Howard E. and Sandewall, Erik, Ed., Interactive Programming Environments, McGraw-Hill Book Co., New York, 1984, pp. 128-140.

12. Donzeau-Gouge, Veronique, Kahn, Gilles, Lang, Bernard and Melese, B. Documents structure and modularity in Mentor. SIGSoft/SIGPlan Software Engineering Symposium on Practical Software Development Environments, April, 1984, pp. 141-148.

13. Engels, G., Gall, R., Nagl, M. and Schafer, W. "Software specification using graph grammars". Computing 31 (1983), 317-346.

14. Farrow, Rodney. "Generating a production compiler from an attribute grammar". IEEE Software 1, 4 (October 1984).

15. Farrow, Rodney. Automatic generation of fixed-point-finding evaluators for circular, but well-defined, attribute grammars. SIGPlan '86 Symposium on Compiler Construction, June, 1986, pp. 85-98.

16. Feiler, Peter H. and Medina-Mora, Raul. "An incremental programming environment". IEEE Transactions on Software Engineering SE-7, 5 (September 1981), 472-482.

17. Feiler, Peter H. LOIPE a language-oriented interactive programming environment based on compilation technology. Ph.D. Th., Carnegie Mellon University, May 1982. CMU-CS-82-117..

18. Feiler, Peter H., Jalili, Fahimeh and Schlichter, Johann H. An interactive prototyping environment for language design. 19th Hawaii International Conference on System Sciences, January, 1986, pp. 106-116.

19. Freeman, Peter. "A conceptual analysis of the Draco approach to constructing software systems". IEEE Transactions on Software Engineering SE-13, 7 (July 1987), 830-844.

20. Ganzinger, Harald, Ripken, Knut and Wilhelm, Reinhard. Automatic generation of optimizing multipass compilers. Information Processing 77, New York, 1977, pp. 535-540. 
21. Garlan, David. Flexible unparsing in a structure editing environment. Tech. Rept. CMUCS-85-129, Carnegie Mellon University, Department of Computer Science, April, 1985.

22. Garlan, David. Views for tools in integrated environments. Ph.D. Th., Carnegie Mellon University, May 1987. CMU-CS-87-147..

23. Ghezzi, Carlo and Mandrioli, Dino. "Augmenting parsers to support incrementality". Journal of the ACM 27, 3 (July 1980), 564-579.

24. Habermann, A. N. and Notkin, D. "Gandalf: software development environments". IEEE Transactions on Software Engineering SE-12, 12 (December 1986), 1117-1127.

25. Henderson, Peter and Weiser, Mark. Continuous execution: the VisiProg environment. 8th International Conference on Software Engineering, August, 1985, pp. 68-74.

26. Hoover, Roger and Teitelbaum, Tim. Efficient incremental evaluation of aggregate values in attribute grammars. SIGPlan '86 Symposium on Compiler Construction, June, 1986, pp. 39-50.

27. Hoover, Roger. Dynamically bypassing copy rule chains in attribute grammars. 13th Annual ACM Symposium on Principles of Programming Languages, January, 1986, pp. 14-25.

28. Horwitz, Susan and Teitelbaum, Tim. "Generating editing environments based on relations and attributes". ACM Transactions on Programming Languages and Systems 8, 4 (October 1986), 577-608.

29. Hudson, Scott E. and King, Roger. Implementing a user interface as a system of attributes. SIGSoft/SIGPlan Software Engineering Symposium on Practical Software Development Environments, December, 1986, pp. 143-149.

30. Jazayeri, M., Ogden, W. F. and Rounds, W. C. "The intrinsically exponential complexity of the circularity problem for attribute grammars". Communications of the ACM 18, 12 (December 1975).

31. Johnson, S. C. and Lesk, M. E. "Language development tools". The Bell System Technical Journal 57, 6 (July-August 1978), 2155-2175.

32. Johnson, Gregory F. and Fischer, Charles N. Non-syntactic attribute flow in language based editors. 9th Annual ACM Symposium on Principles of Programming Languages, January, 1982, pp. 185-195.

33. Johnson, Gregory F. and Fischer, C. N. A meta-language and system for nonlocal incremental attribute evaluation in language-based editors. 12th Annual ACM Symposium on Principles of Programming Languages, January, 1985, pp. 141-151.

34. Johnson, Gregory F. GL — a denotational testbed with continuations and partial continuations as first-class objects. SIGPlan '87 Symposium on Interpreters and Interpretive Techniques, June, 1987, pp. 165-176.

35. Gail E. Kaiser and Elaine Kant. "Incremental Parsing Without A Parser". The Journal of Systems and Software 5, 2 (May 1985), 121-144.

36. Kaiser, Gail E. Semantics of structure editing environments. Ph.D. Th., Carnegie Mellon University, May 1985. CMU-CS-85-131.. 
37. Kaiser, Gail E. and Garlan, David. "Melding software systems from reusable building blocks". IEEE Software (July 1987), 17-24.

38. Kaiser, Gail E., Kaplan, Simon M. and Micallef, Josephine. "Multiuser, distributed language-based environments". IEEE Software (November 1987), 58-67.

39. Gail E. Kaiser, Peter H. Feiler, Fahimeh Jalili and Johann H. Schlichter. "A Retrospective on DOSE: An Interpretive Approach to Structure Editor Generation". Software - Practice \& Experience 18, 8 (August 1988), 733-748.

40. Kaiser, Gail E. and Kaplan, Simon M. Rapid prototyping of concurrent programming languages. 8th International Conference on Distributed Computing Systems, June, 1988, pp. 250-255.

41. Kaiser, Gail E. Concurrent Meld. Workshop on Object-Based Concurrent Programming, September, 1988. To appear.

42. Kastens, U., Hutt, B. and Zimmermann, E.. Lecture Notes in Computer Science. Volume 141:GAG: A Practical Compiler Generator. Springer-Verlag, Heidelberg, 1982.

43. Knuth, Donald E. "Semantics of context-free languages". Mathematical Systems Theory 2, 2 (June 1968), 127-145.

44. Lamb, David Alex . "IDL: sharing intermediate representations". ACM Transactions on Programming Languages and Systems 9, 3 (July 1987), 297-318.

45. Medina-Mora, Raul. Syntax-directed editing: towards integrated programming environments. Ph.D. Th., Carnegie Mellon University, March 1982. CMU-CS-82-113..

46. Notkin, David S. Interactive structure-oriented computing. Ph.D. Th., Carnegie Mellon University, February 1984. CMU-CS-84-103..

47. Partsch, H. and Steinbruggen, R. "Program transformation systems". Computing Surveys 15, 3 (September 1983), 199-236.

48. Paulson, Lawrence. A semantics-directed compiler generator. 9th Annual ACM Symposium on Principles of Programming Languages, January, 1982, pp. 224-233.

49. Raskovsky, Martin R. Denotational semantics as a specification of code generators. SIGPlan '82 Symposium on Compiler Construction, June, 1982, pp. 230-244.

50. Reiss, Steven P. Graphical program development with PECAN program development systems. SIGSoft/SIGPlan Software Engineering Symposium on Practical Software Development Environments, April, 1984, pp. 30-41.

51. Reiss, Steven P. An approach to incremental compilation. SIGPlan '84 Symposium on Compiler Construction, June, 1984, pp. 144-156.

52. Reps, Thomas, Teitelbaum, Tim and Demers, Alan. "Incremental context-dependent analysis for language-based editors". ACM Transactions on Programming Languages and Systems 5, 3 (July 1983), 449-477.

53. Reps, Thomas and Teitelbaum, Tim. The Synthesizer Generator. SIGSoft/SIGPlan Software Engineering Symposium on Practical Software Development Environments, April, 1984, pp. 41-48. 
54. Reps, Thomas, Marceau, Carla and Teitelbaum, Tim. Remote attribute updating for language-based editors. 13th Annual ACM Symposium on Principles of Programming Languages, January, 1986, pp. 1-13.

55. Scott, Dana and Strachey, Christopher. Toward a mathematical semantics for computer languages. Tech. Rept. Technical Monograph PRG-6, Oxford University Computing Laboratory, August, 1971.

56. Shebs, Stan and Kessler, Robert. Automatic design and implementation of language datatypes. SIGPlan '87 Symposium on Interpreters and Interpretive Techniques, June, 1987, pp. 26-37.

57. Smith, Douglas R., Kotik, Gordon B. and Westfold, Stephen J. "Research on knowledgebased software environments at Kestrel Institute". IEEE Transactions on Software Engineering SE-11, 11 (November 1985), 1278-1295.

58. Snodgrass, Richard and Shannon, Karen. Lecture Notes in Computer Science. Volume 244: Supporting flexible and efficient tool integration. In Advanced Programming Environments, Conradi, Reidar, Didriksen, Tor M. and Wanvik, Dag H., Eds., Springer-Verlag, Berlin, 1986, pp. 290-313.

59. Teitelbaum, Tim and Reps, Thomas. "The Cornell Program Synthesizer: a syntax-directed programming environment". Communications of the ACM 24, 9 (September 1981), 563-573.

60. Teitelman, Warren and Masinter, Larry. "The Interlisp programming environment". IEEE Computer 14, 4 (April 1981), 25-34.

61. Walz, Janet A. and Johnson, Gregory F. Incremental evaluation for a general class of circular attribute grammars. SIGPlan '88 Conference on Programming Language Design and Implementation, June, 1988, pp. 209-221.

62. Waters, Richard C. "KBEmacs: where's the AI?". The AI Magazine VII, 1 (Spring 1986), 47-56.

63. Wegman, Mark N. Parsing for structural editors. 21st Annual Symposium on Foundations of Computer Science, October, 1980, pp. 320-327. 